А. В. Лакеев, А. В. Данеев, В. А. Русанов. К билинейной дифференциальной реализации континуального пучка траекторных кривых ...

УДК 517. 93, 517.937

DOI: $10.18101 / 2304-5728-2020-1-11-27$

\title{
К БИЛИНЕЙНОЙ ДИФФЕРЕНЦИАЛЬНОЙ РЕАЛИЗАЦИИ КОНТИНУАЛЬНОГО ПУЧКА ТРАЕКТОРНЫХ КРИВЫХ В КОНСТРУКЦИЯХ ОПЕРАТОРА РЕЛЕЯ - РИТЦА ${ }^{1}$
}

\author{
(C) Лакеев Анатолий Валентинович \\ доктор физико-математических наук, \\ ведущий научный сотрудник, \\ Институт динамики систем и теории управления \\ им. В. М. Матросова \\ Россия, 664033, г. Иркутск, ул. Лермонтова, 134 \\ E-mail: lakeyev@icc.ru \\ (C) Данеев Алексей Васильевич \\ доктор технических наук, профессор, \\ Иркутский государственный университет путей сообщения \\ Россия, 664074, г. Иркутск, ул. Чернышевского, 15 \\ E-mail: daneev@mail.ru \\ (C) Русанов Вячеслав Анатольевич \\ доктор физико-математических наук, \\ старший научный сотрудник, \\ Институт динамики систем и теории управления \\ им. В. М. Матросова \\ Россия, 664033, г. Иркутск, ул. Лермонтова, 134 \\ E-mail: v.rusanov@mail.ru
}

Приводятся функционально-геометрические условия (необходимые и достаточные) существования пяти нестационарных билинейных операторов в модели дифференциальной реализации континуального пучка управляемых траекторных кривых (динамических процессов типа «вход - выход» мощности $\leq \exp \aleph_{0}$ ) в классе билинейных неавтономных обыкновенных дифференциальных уравнений второго порядка (в том числе квазилинейных гиперболических моделей) в вещественном сепарабельном гильбертовом пространстве. Рассматриваемая задача относится к типу нестационарных нелинейных коэффициентных обратных задач для эволюционных уравнений в гильбертовом пространстве и решается на основе качественного изучения свойства непрерывности функционального оператора Релея - Ритца. При этом показано, что структура фундаментальной группы образа данного оператора зависит от размерности проективного пространства, на котором он действует. Полученные результаты имеют приложение к качественной теории нелинейной структурной идентификации полилинейных нестационарных дифференциальных моделей высших порядков.

\footnotetext{
${ }^{1}$ Работа выполнена при финансовой поддержке РФФИ (проекты 19-01-00301, 1908-00746).
} 
Ключевые слова: нелинейные обратные задачи; билинейная нестационарная дифференциальная реализация; оператор Релея — Ритца; фундаментальная группа Пуанкаре.

\section{Для цитирования:}

Лакеев A. B., Данеев А. В., Русанов В. А. К билинейной дифференциальной реализации континуального пучка траекторных кривых в конструкциях оператора Релея - Ритца // Вестник Бурятского государственного университета. Математика, информатика. 2020. № 1. С. 11-27.

\section{Введение}

Поскольку во многих практически важных задачах реализации дифференциального представления моделируемых динамических процессов необходимо учитывать нестационарную нелинейную взаимосвязь как от самой траектории и скорости движения на ней, так и от программного управления, то ниже основное внимание сосредоточено на исследовании модели дифференциальной реализации, зависящей от пяти нестационарных билинейных структур. При этом одна из них задана на самой траектории, второй билинейный оператор зависит от траектории и скорости движения по ней, третий билинейный оператор зависит только от скорости движения по этой траектории и два других учитывают эти переменные в связи с влиянием на них программного управления.

В целом современная качественная теория дифференциальной реализации (КТДР), рассматриваемая в духе бесконечномерной постановки обратных задач математической физики, сложнее, интереснее, глубиннее в своих приложениях и очень важна для понимания основных свойств самих дифференциальных моделей. Ее геометрические конструкции могут служить отправными точками современного развития общей (аксиоматической) теории динамических систем, попутно придавая этим конструкциям репутацию полезного математического инструмента в прецизионном апостериорном моделировании сложных бесконечномерных динамических моделей.

Поскольку КТДР изучает вопросы существования аналитического представления (дифференциальных моделей) для семейств динамических процессов типа «вход - выход» (бихевиористических систем Я. Виллемса [1]), то данная статья могла бы иметь название: Как определить, существует ли билинейное дифференциальное уравнение «черного ящика». Поэтому в методологическом плане КТДР можно рассматривать как важный шаг на пути построения общей теории математического моделирования сложных динамических систем в контексте теории идентификации эволюционных уравнений [2] на стыке функционального анализа [3; 4] и теории дифференциальных уравнений в бесконечномерных пространствах [5]. Причем в теоретико-системном анализе непрерывных слабоструктурированных систем до какого-то момента «механический» перенос качественных результатов конечномерной теории реализации на бесконечномерный случай происходит без особых осложнений. Это отно- 
A. В. Лакеев, А. В. Данеев, В. А. Русанов. К билинейной дифференциальной реализации континуального пучка траекторных кривых ...

сится к стационарным/нестационарным дифференциальным моделям первого порядка (параболические уравнения и системы диффузионного типа) в равномерно выпуклых пространствах Гёльдера [6], или сепарабельных гильбертовых пространствах [7; 8].

Существенные аналитические трудности начинаются при переходе к дифференциальной реализации с динамическим порядком выше первого [9], в том числе неноминальном учете структуры гиперболических моделей [10], при представлении уравнений которых нельзя обойтись без учета нелинейности их динамики. В частности, билинейной структуры модели реализации $[11 ; 12]$, на чем и акцентируется основное внимание в данной статье. При этом покажем, что, осуществляя аналитическую связь между проективной геометрией и дифференциальной реализацией моделируемых бесконечномерных динамических процессов, конструкцию проективизации нелинейного функционального оператора Релея -Ритца $[7 ; 8]$ и функционально-геометрический анализ условий его непрерывности удобно формулировать на языке компактных топологических многообразий тензорных произведений гильбертовых пространств в терминах $C W$-комплексов Уайтхеда [13]. Такая качественная теория до конца еще не создана и не так давно не существовало еще и базы для нее. За последние полтора десятилетия положение изменилось [6-12], и можно считать, что теперь такая база существует в качестве теории расширений нестационарных $M_{p}$-операторов [7] (см. также [11;14], где подытожены основные исследования в этой области).

\section{1 Постановка задачи}

Далее $\left(X,\|\cdot\|_{X}\right),\left(Y,\|\cdot\|_{Y}\right),\left(Z,\|\cdot\|_{Z}\right)$ - вещественные сепарабельные гильбертовы пространства (нормы удовлетворяют «условию параллелограмма» [15, с. 47]); ниже используем [3, с. 176] линейную изометрию (сохраняющую норму) $E: Y \rightarrow X$ пространств $Y$ и $X$. Как обычно $L\left(\mathbf{B}^{\prime}, \mathbf{B}^{\prime \prime}\right)$ - банахово пространство (с операторной нормой) линейных непрерывных операторов для банаховых пространств $\mathbf{B}^{\prime}$ и $\mathbf{B}^{\prime \prime}, \mathrm{L}\left(X^{2}, X\right)-$ пространство всех непрерывных билинейных отображений из декартового квадрата $X \times X$ в пространство $X$ (аналогично $\mathrm{L}\left(X^{2}, Z\right)$ ).

Обозначим через $T$ отрезок числовой прямой $R$ с мерой Лебега $\mu$, через $\wp_{\mu}-\sigma$-алгебру всех $\mu$-измеримых подмножеств из $T$, запись $S \underset{\bmod \mu}{\subseteq} Q$ для $S, Q \in \wp_{\mu}$ означает $\mu(S \backslash Q)=0$. Сверх того примем, что $A C^{1}(T, X)-$ множество всех функций $\varphi: T \rightarrow X$, первая производная которых является абсолютно непрерывной функцией (относительно меры $\mu$ ) на интервале $T$. 
Если ниже $\left(\mathbf{B},\|\cdot\|_{\mathbf{B}}\right)$ - некоторое банахово пространство, то через $\mathrm{L}_{p}(T, \mathbf{B}), p \in[1, \infty)$, как обычно, будем обозначать банахово пространство всех классов $\mu$-эквивалентности интегрируемых по Бохнеру [4, с. 137] отображений $f: T \rightarrow \mathbf{B}$ с нормой $\left.\int_{T}\|f(\tau)\|_{\mathbf{B}}^{p} \mu(d \tau)\right)^{1 / p}$, соответственно через $\mathrm{L}_{\infty}(T, \mathbf{B})$ - банахово пространство данных классов с нормой ess $\sup _{T}\|f\|_{\mathbf{B}}$. В означенном контексте условимся, что

$$
\begin{gathered}
\mathbf{L}_{2}:=\mathrm{L}_{2}(T, \mathrm{~L}(X, X)) \times \mathrm{L}_{2}(T, \mathrm{~L}(X, X)) \times \mathrm{L}_{2}(T, L(Y, X)) \times \\
\times \mathrm{L}_{2}\left(T, \mathrm{~L}\left(X^{2}, X\right)\right) \times \mathrm{L}_{2}\left(T, \mathrm{~L}\left(X^{2}, X\right)\right) \times \mathrm{L}_{2}\left(T, \mathrm{~L}\left(X^{2}, X\right)\right) \times \\
\quad \times \mathrm{L}_{2}\left(T, \mathrm{~L}\left(X^{2}, X\right)\right) \times \mathrm{L}_{2}\left(T, \mathrm{~L}\left(X^{2}, X\right)\right), \\
\quad \mathbf{L}^{*}:=L(X, X) \times L(X, X) \times L(Y, X) \times \\
\times \mathrm{L}\left(X^{2}, X\right) \times \mathrm{L}\left(X^{2}, X\right) \times \mathrm{L}\left(X^{2}, X\right) \times \mathrm{L}\left(X^{2}, X\right) \times \mathrm{L}\left(X^{2}, X\right) .
\end{gathered}
$$

Далее считаем, что на временном интервале $T$ фиксировано (возможно, a posteriori) поведение $[1 ; 16]$ исследуемой бихевиористической системы в виде не ограниченного по мощности нелинейного пучка ${ }^{2} N$ управляемых динамических процессов (управляемых траекторных кривых) типа «вход - выход», т. е. формально:

$$
N \subset\left\{(x, u): x \in A C^{1}(T, X), u \in \mathrm{L}_{2}(T, Y)\right\}, \operatorname{Card} N \leq \exp \aleph_{0},
$$

где $(x, u)$ - пара «траектория, программное управление», $\aleph_{0}$ - алеф нуль, ехр $\aleph_{0}$ - континуум, и пусть задана, как инерционно-массовая характеристика моделируемой системы, оператор-функция при второй производной (в модели реализации) от траектории $t \mapsto x(t)$ вида:

$$
\begin{gathered}
\hat{A} \in \mathrm{L}_{\infty}(T, L(X, X)), \\
\mu\{t \in T: \hat{A}(t)=0 \in L(X, X)\}=0 ;
\end{gathered}
$$

при этом считаем, что нарушение условия эквивалентности нормальной системе, а именно:

$$
\mu\{t \in T: \operatorname{Ker} \hat{A}(t)=0 \in X\}=0,
$$

необременительно (см. ниже замечание 1).

Рассмотрим задачу: для фиксированной пары $(N, \hat{A})$ определить необходимые и достаточные условия, выраженные в терминах нелинейного пучка динамических процессов $N$ и оператор-функции $\hat{A}$, существования упорядоченного набора из восьми оператор-функций

$$
\left(A_{1}, A_{0}, B, \mathrm{D}_{1}, \mathrm{D}_{2}, \mathrm{D}_{3}, \mathrm{D}_{4}, \mathrm{D}_{5}\right) \in \mathbf{L}_{2},
$$

\footnotetext{
2 Термин «нелинейный пучок» означает, что для траекторных кривых пучка $a$ priori не предполагается наличие принщипа суперпозищии (когда зависимость выходных величин от входных воздействий суть линейная [25, с. 18]).
} 
A. В. Лакеев, А. В. Данеев, В. А. Русанов. К билинейной дифференциальной реализации континуального пучка траекторных кривых ...

для которого осуществима билинейная дифференциальная реализация (БДР) вида:

$$
\begin{gathered}
\hat{A} d^{2} x / d t^{2}+A_{1} d x / d t+A_{0} x= \\
=B u+\mathrm{D}_{1}(x, x)+\mathrm{D}_{2}(x, d x / d t)+\mathrm{D}_{3}(d x / d t, d x / d t)+ \\
+\mathrm{D}_{4}(E(u), x)+\mathrm{D}_{5}(E(u), d x / d t), \quad \forall(x, u) \in N ;
\end{gathered}
$$

равенство в (1) рассматривается как тождество в $\mathrm{L}_{1}(T, X)$. Если операторы БДР-системы (1) предполагается искать в классе стационарных, то будем их строить в классе непрерывных, т. е.

$$
\left(A_{1}, A_{0}, B, \mathrm{D}_{1}, \mathrm{D}_{2}, \mathrm{D}_{3}, \mathrm{D}_{4}, \mathrm{D}_{5}\right) \in \mathbf{L}^{*} \text {. }
$$

$\mathrm{B}$ связи с означенной математической постановкой отметим, что каждая область математики, как правило, содержит свои ведущие проблемы, которые настолько трудны, что их полное решение даже и не ожидается, но которые стимулируют постоянный поток работ и служат главными вехами на пути прогресса в этой области. В рамках КТДР такой проблемой является проблема классификации непрерывных бихевиористических систем, рассматриваемых так, как если бы они точно совпадали с решениями идеализированных дифференциальных моделей, в том числе высших порядков. В наиболее сильной форме она предполагает классификацию таких систем с точностью до соответствующего класса моделей дифференциальной реализации, в частности, и класса нестационарных БДР-моделей (1), обоснованием чего ниже служат теорема 1 и ее следствие 1 , которые позволяют в означенной классификации значительно приблизиться к идеальному сочетанию функциональной прозрачности и геометрической наглядности (см. также [16-20]).

\section{2 Существование БДР-модели}

Опишем теперь аналитическую схему решения вопроса о разрешимости (или неразрешимости) БДР-задачи (1). Итак, пусть $Z:=X \otimes X-$ пополненное гильбертово тензорное произведение [15, с. 54] гильбертовых пространств $X$ и $X$ с кросс-нормой $\|\cdot\|_{Z}$, определяемой внутренним произведением [4, с. 64]. Сверх того, примем следующие обозначения:

$$
\begin{gathered}
U:=X \times X \times Y \times Z \times Z \times Z \times Z \times Z, \\
\|(\cdot, \cdot, \cdot, \cdot)\|_{U}:=\left(\|\cdot\|_{X}^{2}+\|\cdot\|_{X}^{2}+\|\cdot\|_{Y}^{2}+\|\cdot\|_{Z}^{2}+\|\cdot\|_{Z}^{2}+\|\cdot\|_{Z}^{2}+\|\cdot\|_{Z}^{2}+\|\cdot\|_{Z}^{2}\right)^{1 / 2} ; \\
\mathrm{M}_{2}:=\mathrm{L}_{2}(T, L(X, X)) \times \mathrm{L}_{2}(T, L(X, X)) \times \mathrm{L}_{2}(T, L(Y, X)) \times \\
\times \mathrm{L}_{2}(T, L(Z, X)) \times \mathrm{L}_{2}(T, L(Z, X)) \times \mathrm{L}_{2}(T, L(Z, X)) \times \\
\times \mathrm{L}_{2}(T, L(Z, X)) \times \mathrm{L}_{2}(T, L(Z, X)) ;
\end{gathered}
$$

ясно, что функциональное пространство $\mathrm{M}_{2}$ (с топологией произведения) линейно гомеоморфно банахову пространству $\mathrm{L}_{2}(T, L(U, X))$. 
Обозначим через $\pi$ универсальное билинейное отображение

$$
\pi: X \times X \rightarrow X \otimes X
$$

на языке категорий морфизм $\pi$ определяет тензорное произведение как универсальный отталкивающий объект $[15$, с. 40$]$. Универсальность билинейного отображения $\pi$ состоит также в том, что

$$
\begin{gathered}
\pi: X \times X \rightarrow X \otimes X \\
\left(\mathrm{x}_{1}, \mathrm{x}_{2}\right) \mapsto \pi\left(\mathrm{x}_{1}, \mathrm{x}_{2}\right)=\mathrm{x}_{1} \otimes \mathrm{x}_{2},\left\|\mathrm{x}_{1} \otimes \mathrm{x}_{2}\right\|_{Z}=\left\|\mathrm{x}_{1}\right\|_{X}\left\|\mathrm{x}_{2}\right\|_{X} ;
\end{gathered}
$$

данные соотношения важны для определения конструкции нелинейного функционального оператора Релея - Ритца (2) в части конкретизации нормы $\|\cdot\|_{U}$.

Далее считаем, что декартов квадрат $X^{2}=X \times X$ наделен нормой $\left(\|\cdot\|_{X}^{2}+\|\|_{X}^{2}\right)^{1 / 2}$. В данной постановке имеет место $\pi \in \mathrm{L}\left(X^{2}, Z\right)$ и, с учетом теоремы 2 [3, с. 245], для любого билинейного отображения $\mathrm{D} \in \mathrm{L}\left(X^{2}, X\right)$ всегда найдется линейный непрерывный оператор $\mathrm{D} \in L(Z, X)$, такой, что справедливо равенство $\mathrm{D}=\mathrm{D} \circ \pi$, при этом для любой пары $(x, u) \in N$ будут выполняться включения:

$$
\begin{gathered}
\pi(x, x), \pi(x, d x / d t), \pi(d x / d t, d x / d t) \in \mathrm{L}_{\infty}(T, Z), \\
\pi(E(u), x), \pi(E(u), d x / d t)) \in \mathrm{L}_{2}(T, Z) .
\end{gathered}
$$

Данные построения подытоживает следующее утверждение.

Лемма 1. Для любого набора $\left(A_{1}, A_{0}, B, \mathrm{D}_{1}, \mathrm{D}_{2}, \mathrm{D}_{3}, \mathrm{D}_{4}, \mathrm{D}_{5}\right) \in \mathbf{L}_{2}$ и отображения

$$
\begin{gathered}
F: \mathrm{L}_{2}(T, X) \times \mathrm{L}_{2}(T, X) \times \mathrm{L}_{2}(T, Y) \times \\
\times \mathrm{L}_{2}\left(T, X^{2}\right) \times \mathrm{L}_{2}\left(T, X^{2}\right) \times \mathrm{L}_{2}\left(T, X^{2}\right) \times \mathrm{L}_{2}\left(T, X^{2}\right) \times \mathrm{L}_{2}\left(T, X^{2}\right) \rightarrow \mathrm{L}_{1}(T, X), \\
\left(y_{1}, y_{2}, y_{3}, y_{4}, y_{5}, y_{6}, y_{7}, y_{8}\right) \mapsto F\left(y_{1}, y_{2}, y_{3}, y_{4}, y_{5}, y_{6}, y_{7}, y_{8}\right):= \\
=A_{1} y_{1}+A_{0} y_{2}+B y_{3}+\mathrm{D}_{1} y_{4}+\mathrm{D}_{2} y_{5}+\mathrm{D}_{3} y_{6}+\mathrm{D}_{4} y_{7}+\mathrm{D}_{5} y_{8}
\end{gathered}
$$

существуют единственный кортеж оператор-функций

$$
\left(D_{1}, D_{2}, D_{3}, D_{4}, D_{5}, D_{6}, D_{7}, D_{8}\right) \in \mathrm{M}_{2}
$$

и, соответственно, единственное линейное отображение

$$
M: \mathrm{L}_{2}(T, U) \rightarrow \mathrm{L}_{1}(T, X),
$$

имеющее аналитическое представление вида

$$
\begin{gathered}
\left(z_{1}, z_{2}, z_{3}, z_{4}, z_{5}, z_{6}, z_{7}, z_{8}\right) \mapsto M\left(z_{1}, z_{2}, z_{3}, z_{4}, z_{5}, z_{6}, z_{7}, z_{8}\right):= \\
=D_{1} z_{1}+D_{2} z_{2}+D_{3} z_{3}+D_{4} z_{4}+D_{5} z_{5}+D_{6} z_{6}+D_{7} z_{7}+D_{8} z_{8},
\end{gathered}
$$

такое, что выполняется следующее функциональное равенство:

$$
\begin{gathered}
\left(y_{1}, y_{2}, y_{3}, y_{4}, y_{5}, y_{6}, y_{7}, y_{8}\right) \mapsto F\left(y_{1}, y_{2}, y_{3}, y_{4}, y_{5}, y_{6}, y_{7}, y_{8}\right)= \\
=M\left(y_{1}, y_{2}, y_{3}, \pi\left(y_{4}\right), \pi\left(y_{5}\right), \pi\left(y_{6}\right), \pi\left(y_{7}\right), \pi\left(y_{8}\right)\right),
\end{gathered}
$$


A. В. Лакеев, А. В. Данеев, В. А. Русанов. К билинейной дифференциальной реализации континуального пучка траекторных кривых ...

которое, в свою очередь, индуцирует для оператор-функций из конструкций отображений $F$ и $M$ следующие операторные соотношения:

$$
A_{1}=D_{1}, A_{0}=D_{2}, B=D_{3} \text {, }
$$

$$
\mathrm{D}_{1}=D_{4} \circ \pi, \mathrm{D}_{2}=D_{5} \circ \pi, \mathrm{D}_{3}=D_{6} \circ \pi, \mathrm{D}_{4}=D_{7} \circ \pi, \mathrm{D}_{5}=D_{8} \circ \pi \text {. }
$$

Следующая лемма обобщает следствие 1 [16].

Лемма 2. Пусть $S, Q \in \wp_{\mu}$, тогда $S \underset{\bmod \mu}{\subseteq} Q$, если имеют место равенства:

$$
\begin{aligned}
S:= & \{t \in T:(g(t), w(t), v(t), q(t), s(t), h(t), \hat{u}(t), \widetilde{u}(t))=0 \in U\}, \\
Q:= & \{t \in T: \dot{g}(t)=0 \in X\}, \dot{g}=d g / d t,(g, w, v, q, s, h, \hat{u}, \tilde{u}) \in V_{N}, \\
V_{N}:= & \operatorname{Span}\{(d x / d t, x, u, \pi(x, x), \pi(x, d x / d t), \pi(d x / d t, d x / d t), \\
& \left.\pi(E(u), x), \pi(E(u), d x / d t)) \in \mathrm{L}_{2}(T, U):(x, u) \in N\right\} .
\end{aligned}
$$

Далее, пусть $\mathrm{L}_{+}(T, R)$ - выпуклый конус [4, с. 127] классов $\mu$ эквивалентности всех вещественных неотрицательных $\mu$-измеримых на интервале $T$ функций и $\leq_{\mathrm{L}}$ - такое квазиупорядочение в пространстве $\mathrm{L}_{+}(T, R)$, что $\xi^{\prime} \leq_{L} \xi^{\prime \prime}$ в том и только в том случае, если $\xi^{\prime}(t) \leq \xi^{\prime \prime}(t) \mu-$ почти всюду в $T$. При этом для заданного подмножества $W \subset \mathrm{L}_{+}(T, R)$ через $\sup _{\mathrm{L}} W$ обозначим наименьшую верхнюю грань подмножества $W$, если эта грань существует в конусе $\mathrm{L}_{+}(T, R)$ в структуре квазиупорядочения $\leq_{\mathrm{L}}$, в частности, имеют место соотношения

$$
\sup _{\mathrm{L}}\left\{\xi^{\prime}, \xi^{\prime \prime}\right\}=\xi^{\prime} \vee \xi^{\prime \prime}:=2^{-1}\left(\xi^{\prime}+\xi^{\prime \prime}+\left|\xi^{\prime}-\xi^{\prime \prime}\right|\right) \text {. }
$$

В данной постановке рассмотрим решетку с ортодополнением [4, c. 339]:

$$
R(W):=\left\{\xi \in \mathrm{L}_{+}(T, R): \xi \leq_{\mathrm{L}} \sup _{\mathrm{L}} W\right\} .
$$

Тогда $\left(R(W), \leq_{\mathrm{L}}\right)$ - решетка с наименьшим $\chi_{\varnothing} \in \mathrm{L}_{+}(T, R)$ и наибольшим $\sup _{\mathrm{L}} W \in \mathrm{L}_{+}(T, R)$ элементами; здесь и далее $\chi_{\varnothing}$ - «нульфункция» конуса $\mathrm{L}_{+}(T, R)$. В контексте определения 3 [21, с. 504] из теоремы 17 [3, с. 68] и следствия 1 [3, с. 69] несложно извлечь более общее утверждение; ниже $\inf _{\mathrm{L}}-$ наибольшая нижняя $\leq_{\mathrm{L}}$-грань.

Лемма 3. Функииональная решетка $R(W)-$ полная, т. е.

$$
\inf _{\mathrm{L}} V, \sup _{\mathrm{L}} V \in \mathbb{R}(W) \forall V \subseteq \mathbb{R}(W) .
$$

Пусть $\Psi: V_{N} \rightarrow \mathrm{L}_{+}(T, R)$ - оператор Релея - Ритца [7; 8]:

$$
t \mapsto \Psi(\varphi)(t):=\left\{\begin{array}{l}
\|\hat{A}(t) \dot{g}(t)\|_{X} /\|\varphi(t)\|_{U}, \text { если } \varphi(t) \neq 0 \in U ; \\
0 \in R, \text { если } \varphi(t)=0 \in U ;
\end{array}\right.
$$

где $\varphi:=(g, w, v, q, s, h, \hat{u}, \tilde{u}) \in V_{N}$. 
Ясно, что имеет место равенство

$$
\begin{gathered}
\|(g(t), w(t), v(t), q(t), s(t), h(t), \hat{u}(t), \tilde{u}(t))\|_{U}:= \\
=\left(\|g(t)\|_{X}^{2}+\|w(t)\|_{X}^{2}+\|v(t)\|_{Y}^{2}+\|q(t)\|_{Z}^{2}+\|s(t)\|_{Z}^{2}+\|h(t)\|_{Z}^{2}+\|\hat{u}(t)\|_{Z}^{2}+\|\tilde{u}(t)\|_{Z}^{2}\right)^{1 / 2} .
\end{gathered}
$$

В силу леммы 2 на интервале времени $T$ выполняется

$$
\operatorname{supp} \Psi(\varphi)=\operatorname{supp}\|\hat{A} \dot{g}\|_{X}(\bmod \mu) ;
$$

здесь в определении supp-конструкции носителя функции следуем [3, c. 137].

Нелинейный оператор (2) удовлетворяет весьма простым (но важным) соотношениям

$$
\chi_{\varnothing} \leq_{\mathrm{L}} \Psi(\varphi)=\Psi(r \varphi), \quad r \in R^{*}:=R \backslash\{0\}, \varphi \in V_{N} ;
$$

ниже будем различать в обозначениях образ точки $\Psi(\varphi)$ и образ множества $\Psi[\{\varphi\}]$.

Теория оператора Релея - Ритца нуждается в точном функциональногеометрическом языке, что заставляет нас уделить этому языку особое внимание. Поэтому прежде чем идти дальше, нам будет удобно ввести дополнительную терминологию. А именно, в силу соотношений (3) оператор $\Psi$ индуцирует отображение $P \Psi: P_{N} \rightarrow \mathrm{L}_{+}(T, R)$, которое, по сложившейся в теории представлений традиции [15, с. 239], назовем проективизацией оператора Релея - Ритца:

$$
P \Psi(\gamma):=\Psi[\gamma], \gamma \in P_{N}\left(\gamma \subset V_{N}\right),
$$

где $P_{N}$ - вещественное проективное пространство, ассоциированное с линейным многообразием $V_{N}$ (с топологией, индуцированной из пространства $\left.\mathrm{L}_{2}(T, U)\right)$; т. е. $P_{N}$ есть множество орбит мультипликативной группы $R^{*}$, действующей на $V_{N} \backslash\{0\}$. В данной геометрической трактовке ключевым моментом являются топологические свойства пространства $P_{N}, \operatorname{dim} P_{N}<\aleph_{\text {}}$, разумеется, в первую очередь (в контексте теоремы 2) его компактность, в частности, если имеет место $\operatorname{dim} V_{N}=3$, то компактное 2-многообразие $P_{N}$ устроено как лист Мёбиуса, к которому по его границе приклеен круг $\left[13\right.$, с. 162]. Попутно отметим, что на $P_{N}$ можно ввести структуру $C W$-комплекса [13, с. 140], что, в свою очередь, упрощает рассмотрение вопроса о геометрической реализации многообразия $P_{N}$ теорема 9.7 [13, с. 149]. 
A. В. Лакеев, А. В. Данеев, В. А. Русанов. К билинейной дифференциальной реализации континуального пучка траекторных кривых ...

Tеорема 1. Каждое из следующих трех условий влечет за собой два других:

(i) БДР-задача (1) разрешима относительно оператор-функций

$$
\left(A_{1}, A_{0}, B, \mathrm{D}_{1}, \mathrm{D}_{2}, \mathrm{D}_{3}, \mathrm{D}_{4}, \mathrm{D}_{5}\right) \in \mathbf{L}_{2}
$$

(i) $\exists \theta \in \mathrm{L}_{2}(T, R): \Psi(\varphi) \leq_{\mathrm{L}} \theta, \forall \varphi \in V_{N}$;

(iii) $\exists \sup _{\mathrm{L}} P \Psi\left[P_{N}\right]: \sup _{\mathrm{L}} P \Psi\left[P_{N}\right] \in \mathrm{L}_{2}(T, R)$.

При этом для выполнения $\left(A_{1}, A_{0}, B, \mathrm{D}_{1}, \mathrm{D}_{2}, \mathrm{D}_{3}, \mathrm{D}_{4}, \mathrm{D}_{5}\right) \in \mathbf{L}^{*}$ необходимо, чтобы

$$
R\left(P \Psi\left[P_{N}\right]\right) \subset \mathrm{L}_{\infty}(T, R) .
$$

Замечание 1. Теорему 1 можно рассматривать как начальный этап в изучении проблемы, когда пучку управляемых траекторных кривых $N$ от неявного дифференциального уравнения высшего порядка требуется сопоставить явную нестационарную билинейную дифференциальную систему второго порядка с тем же пучком управляемых траекторных кривых $N$.

Доказательство. Будем пользоваться идеями работы [17]. Придерживаясь определения 1 [17], введем в рассмотрение конструкцию $M_{2}$ оператора $M: \mathrm{L}_{2}(T, U) \rightarrow \mathrm{L}_{1}(T, X)$ вида

$$
\begin{gathered}
\exists\left(D_{1}, D_{2}, D_{3}, D_{4}, D_{5}, D_{6}, D_{7}, D_{8}\right) \in \mathrm{M}_{2}: M(g, w, v, q, s, h, \hat{u}, \tilde{u}):= \\
=D_{1} g+D_{2} w+D_{3} v+D_{4} q+D_{5} s+D_{6} h+D_{7} \hat{u}+D_{8} \tilde{u} \\
\forall(g, w, v, q, s, h, \hat{u}, \tilde{u}) \in \mathrm{L}_{2}(T, U) .
\end{gathered}
$$

Остальные детали доказательства с небольшими уточнениями (с учетом леммы 1 , леммы 2 и леммы 3 для решетки $\left.R\left(P \Psi\left[P_{N}\right]\right)\right)$ содержит схема $M_{2}$-продолжимости в форме следствия 2 и теоремы 3 [17], при этом необходимое условие для

$$
\left(A_{1}, A_{0}, B, \mathrm{D}_{1}, \mathrm{D}_{2}, \mathrm{D}_{3}, \mathrm{D}_{4}, \mathrm{D}_{5}\right) \in \mathbf{L}^{*}
$$

устанавливается модификацией доказательства теоремы 3 [7]. Теорема доказана.

Замечание 2. Необходимо отметить, что даже в случае $1<\mathrm{Card} N<\aleph_{0}$ имеет место положение $\operatorname{Card} P_{N}=\exp \aleph_{0}$; но можно показать, что существует (теорема 17 [3, с. 68]) такое счетное множество $G \subset P_{N}$, что если в пространстве $\mathrm{L}_{+}(T, R)$ лежит $\sup _{\mathrm{L}} P \Psi\left[P_{N}\right]$, то вещественную функцию $\zeta:=\sup _{\mathrm{L}} P \Psi\left[P_{N}\right]$ осуществляет следующая sup -конструкция:

$$
t \mapsto \zeta(t)=\sup \{P \Psi(\gamma)(t) \in R: \gamma \in G\} .
$$

Замечание 3. Опираясь на теорему 1 , нетрудно сформулировать аналог теоремы 3 [12], выражающий в терминах углового расстояния (в гильбертовом пространстве) условия существования билинейной системы 
(1), реализующей пучки $N_{1}, N_{2}$, каждый из которых обладает своей БДРреализацией, в том числе в постановке [7; 18], когда моделируемые операторы дифференциальной системы (1) суть стационарные, т. е.

$$
\left(A_{1}, A_{0}, B, \mathrm{D}_{1}, \mathrm{D}_{2}, \mathrm{D}_{3}, \mathrm{D}_{4}, \mathrm{D}_{5}\right) \in \mathbf{L}^{*}
$$

в частности, с оптимальной нормой [17].

В конкретных рассуждениях важен также следующий частный случай:

Следствие 1. Если $\operatorname{dim} V_{N}<\aleph_{0}, \Psi\left[V_{N}\right] \subset \mathrm{L}_{2}(T, R)$ и найдется такое число $p \in[1, \infty)$, что будет

$$
\Psi\left(\varphi_{1}+\varphi_{2}\right) \leq_{\mathrm{L}} p \Psi\left(\varphi_{1}\right)+p \Psi\left(\varphi_{2}\right), \quad\left(\varphi_{1}, \varphi_{2}\right) \in V_{N} \times V_{N},
$$

то БДР-задача (1) разрешима.

(При $p=1$ данное свойство, в контексте квазиупорядочения $\leq_{\mathrm{L}}$, сродни свойству «сублинейности» функциональных операторов [26, с. 400]).

\section{3 Вычисление фундаментальной группы и ориентации образа оператора Релея - Ритца}

В случае компактности проективного многообразия $P_{N}$ (равносильно, $\operatorname{dim} P_{N}<\aleph_{0}$ ) естественно попытаться связать это свойство с задачей построения решетки $R\left(P \Psi\left[P_{N}\right]\right)$ в контексте условий непрерывности проективизации оператора Релея - Ритца; ниже при выборе в теореме 2 метрической структуры в конусе $\mathrm{L}_{+}(T, R)$ прибегли к теоремам $15,16[3, \mathrm{c}$. $65,67]$ (в данной постановке $\mathrm{L}_{+}(T, R)$ - полное сепарабельное метрическое пространство).

Теорема 2. Пусть $\operatorname{dim} P_{N}<\aleph_{0}$ и конус $\mathrm{L}_{+}(T, R)$ наделен топологией, индуцированной сходимостью по мере $\mu$, или, что эквивалентно, инвариантной ${ }^{3}$ метрикой

$$
\rho_{T}\left(f_{1}, f_{2}\right):=\int_{T}\left|f_{1}(\tau)-f_{2}(\tau)\right|\left(1+\left|f_{1}(\tau)-f_{2}(\tau)\right|\right)^{-1} \mu(d \tau), \quad f_{1}, f_{2} \in \mathrm{L}_{+}(T, R) .
$$

Тогда оператор $P \Psi: P_{N} \rightarrow \mathrm{L}_{+}(T, R)$ будет непрерывным, если пучок $N$ таков, что

$$
\forall \varphi \in V_{N} \backslash\{0\}: \operatorname{supp}\|\varphi\|_{U}=T(\bmod \mu)
$$

в частности, если

$$
\forall \gamma \in P_{N}: \operatorname{supp} P \Psi(\gamma)=T(\bmod \mu) .
$$

Отметим, что теорема 2 является развитием теоремы 3 [8], что подтверждает ее методологическую важность в математическом (апостериорном) моделировании сложных динамических систем [7; 8; 17-20]. Пер-

\footnotetext{
${ }^{3}$ Вариант непрерывности оператора Релея - Ритца с неинвариантной метрикой см. в [24].
} 
A. В. Лакеев, А. В. Данеев, В. А. Русанов. К билинейной дифференциальной реализации континуального пучка траекторных кривых ...

вое приложение этого результата (с учетом теоремы 3 [22, с. 61] и следствия 5.3 [23, с. 137]) составляет следующее утверждение, описывающее (в зависимости от $\operatorname{dim} \operatorname{Span} N$ ) геометрическое обустройство образа оператора Релея - Ритца.

Теорема 3. Если при выполнении (4) или (5) оператор $P \Psi$ взаимнооднозначный, то $P \Psi$ - гомеоморфизм, а фундаментальная группа метрического пространства $\left(P \Psi\left[P_{N}\right], \rho_{T}\right)$ изоморфна аддитивной группе целых чисел $\mathrm{Z}$ при $\operatorname{dim} \operatorname{Span} N=2$ и группе вычетов $\mathrm{Z}_{2}$ при $\operatorname{dim} \operatorname{Span} N \geq 3$, причем пространство $\left(P \Psi\left[P_{N}\right], \rho_{T}\right)$ ориентируемо, если размерность линейной оболочки $\operatorname{Span} N$ четная, и не ориентируемо, если эта размерность нечетная.

В завершение проведем рассуждения, иллюстрирующие (в главных чертах) возможный аналитический подход в функциональном анализе пары $(N, \hat{A})$, приводящий к развитию теоремы 2.

Введем в рассмотрение функциональное множество вида:

$$
\mathrm{L}_{\times}(T, R):=\left\{(f, g) \in \mathrm{L}_{+}(T, R) \times \mathrm{L}_{+}(T, R): \operatorname{supp} f \underset{\bmod \mu}{\subseteq} \operatorname{supp} g\right\} ;
$$

$\mathrm{L}_{х}(T, R)$ не является линейным пространством, но таковые содержит, включая бесконечномерные.

Рассмотрим нелинейный функциональный оператор

$$
\psi: \mathrm{L}_{\times}(T, R) \rightarrow \mathrm{L}_{+}(T, R)
$$

с конструкцией вида:

$$
\begin{gathered}
\psi(f, g)(t):=f(t) g^{-1}(t) \text { при } g(t) \neq 0, \\
\psi(f, g)(t):=0 \text { для } g(t)=0 ;
\end{gathered}
$$

с учетом леммы 2 данная конструкция индуцирована оператором (2).

Далее, введем последовательность $\left\{\left(f_{n}, g_{n}\right)\right\} \subset \mathrm{L}_{\times}(T, R)$, для которой справедливо

$$
\rho_{T}\left(f_{n}, f_{m}\right) \rightarrow 0, \quad \rho_{T}\left(g_{n}, g_{m}\right) \rightarrow 0 \quad(n, m \rightarrow \infty) .
$$

Сверх того, с учетом полноты метрического пространства $\left(\mathrm{L}_{+}(T, R), \rho_{T}\right)$, потребуем, чтобы

$$
\left(\operatorname{Lim}_{\rho_{T}}\left\{f_{n}\right\}, \operatorname{Lim}_{\rho_{T}}\left\{g_{n}\right\}\right) \in \mathrm{L}_{\times}(T, R) ;
$$

для удобства обозначим $f:=\operatorname{Lim}_{\rho_{T}}\left\{f_{n}\right\}, g:=\operatorname{Lim}_{\rho_{T}}\left\{g_{n}\right\}$.

В данной постановке для сходимости

$$
\rho_{T}\left(\psi\left(f_{n}, g_{n}\right), \psi(f, g)\right) \rightarrow 0(n \rightarrow \infty)
$$

достаточно, чтобы

$$
\lim \left\{\mu\left(\operatorname{supp} g_{n} \Delta \operatorname{supp} g\right): n \rightarrow \infty\right\}=0,
$$

где $\Delta$ - симметрическая разность $\operatorname{supp} g_{n}$ и supp $g$, т. е. 


\section{( $\left.\operatorname{supp} g_{n} \backslash \operatorname{supp} g\right) \cup\left(\operatorname{supp} g \backslash \operatorname{supp} g_{n}\right)$.}

Хотя условие (6) аналитически интересно и полезно [24], тем не менее оно, к сожалению, не является необходимым ${ }^{4}$, что указывает на определенную «избыточность» условий (4), (5) теоремы 2. В данном контексте остается открытым вопрос о построении характеристического признака для пары $\left(\left\{\left(f_{n}, g_{n}\right)\right\},(f, g)\right)$, определяющего $\rho_{T}$-сходимость вида:

$$
\rho_{T}\left(\psi\left(f_{n}, g_{n}\right), \psi(f, g)\right) \rightarrow 0(n \rightarrow \infty) .
$$

В завершение отметим, что теорема 3 дает дополнительные основания для побудительных мотивов в дальнейшем тополого-алгебраическом изучении БДР-реализации (в рамках КТДР в контексте разработки тензорного основания функционально-геометрической теории нестационарных билинейных векторных полей [23]), в частности, для нелинейных динамических систем, траектории которых суть аналитические функции [27], что a priori обеспечивает условие (4) теоремы 2 в силу принципа изолированности нулей.

\section{Заключение}

Можно констатировать, что как самостоятельная область теории обратных задач математической физики качественная теория дифференциальной реализации в сепарабельном гильбертовом пространстве превратилась в сильно развитую экспансивную науку, представляя собой трудно обозримый конгломерат большого количества разнообразных идей и методов, в высшей степени полезный для задач структурнопараметрической идентификации эволюционных уравнений. К мощным и часто применяемым методам КТДР относится аппарат нелинейных функциональных операторов Релея - Ритца. В данном контексте в работе исследована непрерывность оператора Релея - Ритца относительно сходимости по мере. В целом это позволило качественно продвинуться в изучении тополого-алгебраической структуры образа данного оператора, в частности, в терминах фундаментальной группы Пуанкаре. Подобные изыскания предполагают глубокое проникновение в физическое содержание предмета, руководствуясь идеей, что сам предмет нестационарной полилинейной дифференциальной реализации исходит из аподиктической простоты какого-то высшего рода, по крайней мере если придерживаться точки зрения Поппера 5 .

\footnotetext{
${ }^{4}$ Условие (6) становится необходимым для выполнения сходимости $\rho_{T}\left(\psi\left(f_{n}, g_{n}\right), \psi(f, g)\right) \rightarrow 0(n \rightarrow \infty)$,
}

если функциональную последовательность $\left\{f_{n}\right\}$ обременить дополнительным положением: найдется такое $\delta>0$, для которого $f_{n}(t) \geq \delta$ при любом $n$ и $\mu$ почти всех $t \in \operatorname{supp} g$ (см. замечание 2 [24]).

${ }^{5}$ К. Р. Поппер (логик, представитель аналитической философии) выдвинул принцип фальсификации (опровержимости), согласно которому критерий научности теории определяется возможностью ее опровержения опытом [28, с. 36]. 
A. В. Лакеев, А. В. Данеев, В. А. Русанов. К билинейной дифференциальной реализации континуального пучка траекторных кривых ...

\section{Литература}

1. Willems J. C. System Theoretic Models for the Analysis of Physical Systems // Ric. Aut. 1979. № 10. P. 71-106.

2. Ahmed N. U. Optimization and Identification of Systems Governed by Evolution Equations on Banach Space. New York: John Wiley and Sons, 1988. 187 p.

3. Канторович Л. В., Акилов Г. П. Функциональный анализ. М.: Наука, 1977. $744 \mathrm{c.}$

4. Рид М., Саймон Б. Методы современной математической физики. Т. 1. Функциональный анализ. М.: Мир, 1977. 360 с.

5. Далецкий Ю. Л., Фомин С. В. Меры и дифференциальные уравнения в бесконечномерных пространствах. М.: Наука, 1983. 384 с.

6. Гольдман Н. Л. Определение коэффициентов при производной по времени в квазилинейных параболических уравнениях в пространствах Гёльдера // Дифференциальные уравнения. 2012. Т. 48, № $12 . \quad$ С. 1597-1606 [https://doi.org/10.1134/S0012266112120026].

7. Rusanov V. A., Daneev A. V., Lakeev A. V., Linke Yu. É. On the Differential Realization Theory of Nonlinear Dynamic Processes in Hilbert Space // Far East Journal of Mathematical Sciences. 2015. Vol. 97, № 4. P. 495-532 [http://dx.doi.org/10.17654/FJMSJun2015_495_532].

8. Русанов В. А., Данеев А. В., Линке Ю. Э. К геометрическим основам дифференциальной реализации динамических процессов в гильбертовом пространстве // Кибернетика и системный анализ. 2017. Т. 53, № 4. С. 71-83 [DOI: 10.1007/s10559-017-9957-z].

9. Rusanov V. A., Daneev A. V., Lakeyev A. V., Sizykh V. N. Higher-Order Differential Realization of Polylinear-Controlled Dynamic Processes in a Hilbert Space // Advances in Differential Equations and Control Processes. 2018. Vol. 19, № 3. P. 263-274 [http://dx.doi.org/10.17654/DE019030263].

10. Аниконов Ю. Е., Нещадим М. В. Об аналитических методах в теории обратных задач для гиперболических уравнений // Сибирский журнал индустриальной математики. 2011. Т. 14, № 2. С. 28-33 [https://doi.org/10.1134/S1990478912010024].

11. Rusanov V. A., Banshchikov A. V., Daneev A. V., Lakeyev A. V. Maximum Entropy Principle in the Differential Second-Order Realization of a Nonstationary Bilinear System // Advances in Differential Equations and Control Processes. 2019. Vol. 20, № 2. P. 223-248 [http://dx.doi.org/ 10.17654/DE020020223].

12. Лакеев А. В., Линке Ю. Э., Русанов В. А. К реализации полилинейного регулятора дифференциальной системы второго порядка в гильбертовом пространстве // Дифференциальные уравнения. 2017. Т. 53, № 8. С. 1098-1109 [DOI: 10.1134/S037406411708012X].

13. Прасолов В. В. Элементы комбинаторной и дифференциальной топологии. М.: МЦНМО, 2014. 360 с.

14. Rusanov V. A., Daneev A. V., Lakeev A. V., Linke Yu. É., Vetrov A. A. System-Theoretical Foundation for Identification of Dynamic Systems. II. // Far East Journal of Mathematical Sciences. 2019. Vol. 116, № 1. P. 25-68 [http://dx.doi.org/10.17654/MS116010025].

15. Кириллов А. А. Элементы теории представлений. М.: Наука, 1978. 344 с.

16. Rusanov V. A., Antonova L. V., Daneev A. V. Inverse Problem of Nonlinear Systems Analysis: a Behavioral Approach // Advances in Differential Equations and Control Processes. 2012. Vol. 10, № 2. P. 69-88. 
17. Русанов В. А., Лакеев А. В., Линке Ю. Э. Существование дифференциальной реализации динамической системы в банаховом пространстве в конструкциях расширений до $\mathrm{M}_{\mathrm{p}}$-операторов // Дифференциальные уравнения. 2013. Т. 49, № 3. C. 358-370 [DOI: 10.1134/S0012266113030105].

18. Русанов В. А., Лакеев А. В., Линке Ю. Э. К разрешимости дифференциальной реализации минимального динамического порядка семейства нелинейных процессов «вход - выход» в гильбертовом пространстве // Дифференциальные уравнения. 2015. Т. 51, № 4. C. 524-537 [DOI: 10.1134/S037406411504010X].

19. Chen Y. A New One-Parameter Inhomogeneous Differential Realization of the $\operatorname{spl}(2,1)$ Superalgebra // Intern. Journal of Theoretical Physics. 2012. Vol. 51, № 12. P. 3763-3768 [DOI: 10.1007/s10773-012-1261-0].

20. Русанов В. А., Данеев А. В., Линке Ю. Э. К оптимизации процесса юстировки модели дифференциальной реализации многомерной системы второго порядка // Дифференциальные уравнения. 2019. Т. 55, № 10. С. 1432-1438 [DOI: 10.1134/S0374064119100145].

21. Иосида К. Функциональный анализ. М.: Мир, 1967. 624 с.

22. Фоменко А. Т., Фукс Д. Б. Курс гомотопической топологии. Москва: Наука, 1989. 528 с.

23. Новиков С. П., Тайманов И. А. Современные геометрические структуры и поля. М.: МЦНМО, 2014. 584 с.

24. Лакеев А. В., Линке Ю. Э., Русанов В. А. Об одном критерии непрерывности оператора Релея - Ритца // Вестник Бурятского государственного университета. 2018. Вып. 3. Математика и информатика. С. 3-13 [DOI: 10.18101/23045728-2018-3-3-13].

25. Калман Р., Фалб П., Арбиб М. Очерки по математической теории систем. М.: Мир, 1971. 400 с.

26. Эдвардс Р. Функциональный анализ: теория и приложения. М.: Мир, 1969. $1072 \mathrm{c}$.

27. Громов В. П. Аналитические решения дифференциально-операторных уравнений в локально выпуклых пространствах // Доклады РАН. 2004. Т. 394, № 3. С. 305-308. $486 \mathrm{p}$.

28. Popper K. R. Conjecture and Refutations. London: Harper and Row. 1963.

\section{ON BILINEAR DIFFERENTIAL REALIZATION OF A CONTINUAL BEAM OF TRAJECTORY CURVES IN THE CONSTRUCTIONS OF THE RAYLEIGH- RITZ OPERATOR}

Anatoliy $V$. Lakeyev

Dr. Sci. (Phys. and Math.), Chief Researcher,

Matrosov Institute for System Dynamics and Control Theory SB RAS

134 Lermontova St., Irkutsk 664033, Russia

E-mail: lakeyev@icc.ru

Aleksey V. Daneev

Dr. Sci. (Engineering), Prof.,

Irkutsk State Transport University,

15 Chernyshevsky St., Irkutsk 664074, Russia

E-mail: daneev@mail.ru 
A. В. Лакеев, А. В. Данеев, В. А. Русанов. К билинейной дифференциальной реализации континуального пучка траекторных кривых ...

Vyacheslav A. Rusanov

Dr. Sci. (Phys. and Math.), Chief Researcher,

Matrosov Institute for System Dynamics and Control Theory SB RAS

134 Lermontov St., Irkutsk 664033, Russia

E-mail:v.rusanov@mail.ru

We present functional and geometric conditions (necessary and sufficient) for the existence of five non-stationary bilinear operators in a model of differential realization of a continuous bundle of controlled trajectory curves (dynamic processes of the power input / output type) in the class of the second order bilinear non-autonomous ordinary differential equations (including quasi-linear hyperbolic models) in a real parallel Hilbert space. The problem under consideration is a type of non-stationary nonlinear coefficient inverse problems for evolutionary equations in Hilbert space and is solved on the basis of a qualitative study of the continuity property of the Rayleigh-Ritz functional operator. It is shown that the structure of the fundamental group of the image of this operator depends on the dimension of the projective space on which it acts. The results obtained are applied to the qualitative theory of non-linear structural identification of higher order multilinear non-stationary differential models.

Keywords: nonlinear inverse problems; bilinear non-stationary differential realization; the Rayleigh-Ritz operator; the Poincaré group.

\section{References}

1. Willems J.C. System Theoretic Models for the Analysis of Physical Systems. Ric. Aut. 1979. No. 10. Pp. 71-106.

2. Ahmed N. U. Optimization and Identification of Systems Governed by Evolution Equations on Banach Space. New York: John Wiley and Sons, 1988. 187 p.

3. Kantorovich L. V., Akilov G. P. Funktsionalnyi analiz [Functional Analysis]. Moscow: Nauka Publ., 1977. 744 p.

4. Reed M., Simon B. Methods of Modern Mathematical Physics. Vol. 1. Functional Analysis. New York: Academic Press, 1972. 344 p.

5. Daletsky Yu. L., Fomin S. V. Mery i differentsialnye uravneniya v beskonechnomernykh prostranstvakh [Measures and Differential Equations in InfiniteDimensional Spaces]. Moscow: Nauka Publ., 1983. 384 p.

6. Goldman N. L. Opredelenie koeffitsientov pri proizvodnoi po vremeni v kvazilineinykh parabolicheskikh uravneniyakh v prostranstvakh Geldera [Finding the Coefficient Multiplying the Time Derivative in Quasilinear Parabolic Equations in Hölder spaces]. Differential Equations. 2012. Vol. 48, no. 12. Pp. 1597-1606. DOI: org/10.1134/S0012266112120026].

7. Rusanov V. A., Daneev A. V., Lakeyev A. V., Linké Yu. E. On the Dif-ferential Realization Theory of Nonlinear Dynamic Processes in Hilbert Space. Far East Journal of Mathematical Sciences. 2015. Vol. 97, no. 4. Pp. 495-532. DOI: org/10.17654/FJMSJun2015_495_532].

8. Rusanov V. A., Daneev A. V., Linké Yu. E. K geometricheskim osnovam differentsialnoi realizatsii dinamicheskikh protsessov $\mathrm{v}$ gilbertovom prostranstve [To the Geometrical Theory of Differential Realization of Dynamic Processes in a Hilbert Space]. Cybernetics and Systems Analysis. 2017. Vol. 53, No. 4. Pp. 71-83. DOI: 10.1007/s10559-017-9957-z . 
9. Rusanov V. A., Daneev A. V., Lakeyev A. V., Sizykh V. N. Higher-Order Different Realization of Polyline-Controlled Dynamic Processes in a Hilbert Space. Advances in different Equations and Control Processes. 2018. Vol. 19, no. 3. Pp. 263274. DOI: org/10.17654/DE019030263 ].

10. Anikonov Yu. E., Neshchadim M. V. Ob analiticheskikh metodakh v teorii obratnykh zadach dlya giperbolicheskikh uravnenii [On Analytical Methods in the Theory of Inverse Problems for Hyperbolic Equations]. Journal of Applied and Industrial Mathematics. 2011. Vol. 14, no. 2. Pp. 28-33. DOI: org/10.1134/S1990478912010024.

11. Rusanov V. A., Banshchikov A. V., Daneev A. V., Lakeyev A. V. Maximum Entropy Principle in the Differential Second-Order Realization of a Nonstationary Bilinear System. Advances in Differential Equations and Control Processes. 2019. Vol. 20, no. 2. Pp. 223-248. DOI: org/10.17654/DE020020223.

12. Lakeyev A.V., Linké Yu. E., Rusanov V. A. K realizatsii polilineinogo regulyatora differentsial'noi sistemy vtorogo poryadka $\mathrm{v}$ gilbertovom [Realization of a Polylinear Controller as a Second-Order Differential System in a Hilbert Space]. Differential Equations. 2017. Vol. 53, No. 8. Pp. 1098-1109. DOI: 10.1134/S037406411708012X.

13. Prasolov V. V. Elementy kombinatornoi i differentsialnoi topologii [Elements of Combinatorial and Differential Topology]. Moscow: MCCME Publ., 2014. 360 p.

14. Rusanov V. A., Daneev A. V., Lakeyev A. V., Linké Yu. E., Vetrov A. A. System-Theoretical Foundation for Identification of Dynamic Systems. II. Far East Journal of Mathematical Sciences. 2019. Vol. 116, no. 1. Pp. 25-68 DOI: org/10.17654/MS116010025 ].

15. Kirillov A. A. Elementy teorii predstavlenii [Elements of Representation Theory]. Moscow: Nauka Publ., 1978. 344 p.

16. Rusanov V. A., Antonova L. V., Daneev A. V. Inverse Problem of Nonlinear Systems Analysis: A Behavioral Approach. Advances in Differential Equations and Control Processes. 2012. Vol. 10, no. 2. Pp. 69-88.

17. Rusanov V. A., Lakeyev A. V., Linké Yu. E. Sushchestvovanie differentsialnoi realizatsii dinamicheskoi sistemy $\mathrm{v}$ banakhovom prostranstve $\mathrm{v}$ konstruktsiyakh rasshirenii do $\mathrm{M}_{p}$-operatorov [Existence of a Differential Realization of a Dynamical System in a Banach Space in the Constructions of Extensions to $\mathrm{M}_{p^{-}}$ operators]. Differential Equations. 2013. Vol. 49, no. 3. Pp. 358-370. DOI: 10.1134/S0012266113030105.

18. Rusanov V. A., Lakeyev A. V., Linké Yu. E. K razreshimosti differentsialnoi realizatsii minimalnogo dinamicheskogo poryadka semeistva nelineinykh protsessov "vkhod-vykhod" v gilbertovom prostranstve [Solvability of Differential Realization of Minimum Dynamic Order for a Family of Nonlinear Input-Output Processes in Hilbert Space]. Differential Equations. 2015. Vol. 51, no. 4. Pp. 524-537. DOI: 10.1134/S037406411504010X.

19. Chen Y. A New One-Parameter Inhomogeneous Differential Realization of the $\operatorname{spl}(2,1)$ Superalgebra. Intern. Journal of Theoretical Physics. 2012. Vol. 51, no. 12. P. 3763-3768. DOI: 10.1007/s10773-012-1261-0.

20. Rusanov V. A., Daneev A. V., Linké Yu. E. K optimizatsii protsessa yustirovki modeli differentsialnoi realizatsii mnogomernoi sistemy vtorogo poryadka [Adjustment Optimization for a Model of Differential Realization of a Multidimensional SecondOrder System]. Differential Equations. 2019. Vol. 55, no. 10. Pp. 1432-1438. DOI: 10.1134/S0374064119100145.

21. Yoshida K. Functional Analysis. Springer Berlin Heidelberg, 1966. 458 p. 
A. В. Лакеев, А. В. Данеев, В. А. Русанов. К билинейной дифференциальной реализации континуального пучка траекторных кривых ...

22. Fomenko A. T., Fuks D. B. Kurs gomotopicheskoi topologii [A Course in Homotopic Topology]. Moscow: Nauka Publ., 1989, 528 p.

23. Novikov S. P., Taimanov I. A. Sovremennye geometricheskie struktury i polya [Modern Geometric Structures and Fields]. Moscow: MCCME Publ., 2014. 584 p.

24. Lakeyev A.V., Linké Yu. E., Rusanov V. A. On a Criterion for the Continuity of The Rayleigh-Ritz Operator. Vestnik Buryatskogo gosudarstvennogo universiteta. Matematika i informatika. 2018. Vyp. 3. Pp. 3-13. DOI: 10.18101/2304-5728-2018-33-13.

25. Kalman P. L. Falb \& M. A. Arbib. Topics in Mathematical System Theory. New York: McGraw-Hill, 1969. 358 p.

26. Edwards R. Functional Analysis: Theory and Applications. New York: Holt, Rinehart and Winston, 1965. $781 \mathrm{p}$.

27. Gromov V. P. Analiticheskie resheniya differentsialno-operatornykh uravnenii v lokalno vypuklykh prostranstvakh [Analytical Solutions for Differential Operator Equations in Locally Convex Spaces]. Doklady Mathematics. 2004. Vol. 394, no. 3. Pp. 305-308.

28. Popper K. R. Conjecture and Refutations. London: Harper and Row, 1963. 486 p. 